\title{
Miranda
}

Revue pluridisciplinaire du monde anglophone /

Multidisciplinary peer-reviewed journal on the English-

speaking world

17 | 2018

Paysages et héritages de David Bowie

\section{Françoise Clary, Plus sur Caryl Phillips. Includes exclusive interviews with Caryl Phillips}

\section{Christine Dualé}

\section{OpenEdition}

Journals

Édition électronique

URL : http://journals.openedition.org/miranda/15129

DOI : 10.4000/miranda.15129

ISSN : 2108-6559

Éditeur

Université Toulouse - Jean Jaurès

\section{Référence électronique}

Christine Dualé, «Françoise Clary, Plus sur Caryl Phillips. Includes exclusive interviews with Caryl

Phillips », Miranda [En ligne], 17 | 2018, mis en ligne le 02 octobre 2018, consulté le 16 février 2021

URL : http://journals.openedition.org/miranda/15129; DOI : https://doi.org/10.4000/miranda.15129

Ce document a été généré automatiquement le 16 février 2021.

\section{cc) (i) () $\Theta$}

Miranda is licensed under a Creative Commons Attribution-NonCommercial-NoDerivatives 4.0 International License. 


\title{
Françoise Clary, Plus sur Caryl Phillips. Includes exclusive interviews with Caryl Phillips
}

\author{
Christine Dualé
}

\section{RÉFÉRENCE}

Françoise Clary, Plus sur Caryl Phillips. Includes exclusive interviews with Caryl Phillips.

Collection Les Clefs concours Anglais-Littérature dirigée par Elisabeth Soubrenie (Paris :

Atlande, 2018), 190 p, ISBN : 978-2-35030-492-2.

1 En 2017, dans Caryl Phillips, Crossing the River, Françoise Clary nous avait livré une première analyse particulièrement riche de l'œuvre maitresse de Caryl Phillips ; elle poursuit son travail et sa réflexion avec ce second ouvrage où Caryl Phillips s'exprime directement. La parole de l'écrivain est en effet le pivot central de l'ouvrage et les divers entretiens consacrés à Françoise Clary sont un moyen supplémentaire, pour le lecteur, de comprendre les questionnements autour de l'altérité, de la transmission du passé et de la différence culturelle. Phillips souligne d'ailleurs : "the journey back to one's past is the beginning of self-knowledge" (178).

2 Ce second ouvrage proposé par Françoise Clary présente trois parties qui s'articulent autour de la transmission de la mémoire (à travers les notions d'espace et de temps) et autour de la réécriture de l'histoire de la diaspora noire par Phillips. La fragmentation et la dislocation sont les maîtres-mots qui définissent la diaspora noire, son histoire et sa mémoire et c'est cette écriture du trauma, ou plutôt cette réécriture, qui apparaît en filigrane des échanges entre Françoise Clary et Caryl Phillips et est ainsi prétexte à préciser et approfondir la pensée de l'écrivain.

3 Cet ouvrage va bien au-delà d'une simple restitution d'entretiens entre l'auteur et Caryl Phillips puisque chacune des trois parties («La transmission polyphonique de la mémoire » (21-44) ; «De la contre-modernité coloniale au modernisme cosmopolite » 
(45-113) ; "Identité et altérité» (115-154) offre une analyse riche et détaillée permettant une mise en perspective du contexte culturel. L'analyse que propose Françoise Clary, à la lumière des propos de Phillips, est aussi un moyen d'approfondir le rapport entre écriture et histoire soulignant ainsi la vision de l'histoire depuis la marge et « la marginalisation culturelle, générée par l'aliénation coloniale et donc source de l'ambivalence identitaire » (157). Comme l'auteure l'écrit elle-même à propos de son ouvrage, «il s'apparente à un faisceau de perspectives destiné à projeter un nouvel éclairage sur Crossing the River pour en faire ressortir le modernisme à travers un choix de thématiques complémentaires ». Encore une fois, de multiples pistes de réflexion historique et esthétique sont proposées ici. En annexes, Françoise Clary présente des informations additionnelles notamment «comment expliquer l'écriture révolutionnaire par le refus des normes " (155) autour d'une narration «historicofictionnelle » fragmentée et un texte qui «entraîne le lecteur vers les interstices de l'histoire » (155). L'ouvrage se clôt sur les huit entretiens réalisés entre juillet et décembre 2017.

texte et les objectifs de Phillips font écho à ceux d'un autre auteur: V.S. Naipau pour qui passé et présent sont aussi étroitement liés dans l'interprétation de l'expérience diasporique. En effet, ces deux auteurs partagent la même exigence: «transformer l'histoire, refaçonner le monde et, ce faisant, comparer et classer les phénomènes sociaux [...] pour étudier la réalité du passé, retranscrire l'histoire à travers le prisme de l'imagination, mais aussi interpréter la signification des faits par le biais de l'expérience diasporique » (35). «Le refus d'inscrire la mémoire africaine dans la même appartenance que la mémoire euro-centrique " (83) est fondamental pour Phillips. La mémoire et sa transmission font donc partie intégrante de son écriture: « memory is critically important to how I think and what I write about» (77). Aussi la transmission de la mémoire passe par une réinterprétation chez Phillips qui propose ainsi une vision postmoderne et postcoloniale de l'histoire de la diaspora noire en déconstruisant et en mettant en péril les normes traditionnelles établies. Comme le souligne Anne Garrait-Bourrier dans son article The Destruction and Impossible Reconstruction of Family Links in 'Crossing the River' by Caryl Phillips (Revue Commonwealth Essays and Studies - Caryl Phillips (vol. 40.1) : 2018, 172 p), la figure patriarcale décrite par Phillips dans Crossing the River est intimement liée à l'histoire et à la déconstruction historique et mémorielle (Garrait-Bourier 46).

Phillips s'interroge non seulement sur l'identité diasporique mais surtout sur la façon dont la mémoire culturelle a pu se transmettre. En explorant l'identité et la mémoire diasporiques, il place le mouvement et la fluidité au cœur de son écriture ce qui pose la question de la fixité des normes et de leur (dé)construction par l'écrivain pour qui la traversée (crossing the river) s'apparente à une stratégie de reconstruction identitaire (Garrait-Bourrier 46). Bénédicte Ledent précise d'ailleurs dans Caryl Phillips (2002): "The multiple physical and cultural passages experienced by all the characters constantly challenge the roles traditionally imposed upon them by class, race, gender or nation. As each metaphorical river-crossing leads to a new translation of the self, we are made to ponder upon the subjectivity, relativity or inadequacy of such categories" (Ledent 58).

6 En plaçant son roman dans une perspective résolument postcoloniale, Caryl Phillips invite le lecteur à ne pas envisager une fixité identitaire dans le processus d'hybridité. Le mouvement et le déplacement sont effectivement essentiels dans l'écriture de 
Phillips qui propose "une relecture mobile et créative de l'histoire de la diaspora africaine " (Garrait-Bourrier 50). Au-delà de cette fluidité, l'écriture de Phillips bouscule et subvertit les normes. En ce sens sa vision s'apparente aux concepts d'« appareil d'état » ou encore de " machine de guerre » de la philosophie deleuzienne. Ces concepts imposent, en effet, une forme de contrôle spécifique fondée sur la binarité et la symétrie. Les sociétés occidentales sont organisées autour d'oppositions, des binarités qui empêchent toute fluidité et, au contraire, imposent un mode de pensée fixe et difficilement transformable. À ces binarités fixes et immuables, Gilles Deleuze oppose le mouvement, la fluidité, l'individu dans sa singularité, son dynamisme et sa subjectivité. Ces assemblages forment des intensités, des variations qui s'opposent constamment à «l'espace contrôlé » pour former des "machines de guerre ». Par conséquent, «l'appareil d'État» est organisé autour d'un langage et d'un contenu spécifiques dominés par une vision masculine blanche occidentale. Aussi, en mettant potentiellement en péril ces oppositions identitaires fixes et établies, Phillips interroge l'histoire et la mémoire culturelle diasporique afin de livrer une nouvelle historicité et de mettre en évidence « une vision sociologique de l'histoire» (44) comme il le confie d'ailleurs à Françoise Clary : "I'm not sure that I would know how to look at the past except through the 'socio-realistic' lens. [...] The events of the past are usually presented in a socio-realistic manner" (44).

7 Enfin, ce qui se dégage de la lecture de l'œuvre de Phillips mais aussi de sa pensée telle qu'il l'a livrée à Françoise Clary, c'est la portée d'une écriture liée à la «valeur collective " de l'énonciation et au chevauchement entre individuel et collectif. En reformulant l'histoire de la diaspora noire, le passé est réinvesti dans Crossing the River et Colour me English comme pour mieux réconcilier la conscience individuelle et collective de l'histoire et du passé. En cela, le schéma proposé par Phillips s'apparente au concept de "remémoration" (rememory) utilisé par Toni Morrison et développé dans Beloved. La perspective de Caryl Phillips renforce le renouvellement créatif d'une écriture et d'une culture de la marge qui vise à exprimer une ambivalence identitaire, une identité fragmentée, "négociée ", pour reprendre la théorie postcoloniale d'Homi Bhabha, et par conséquent «une vérité hybride» (46) qui s'est construite dans cet « interstice », cet « entre-deux» définis par Homi Bhabha dans The Location of Culture. Que ce soit à travers Crossing the River ou Colour me English, Caryl Phillips ouvre à une lecture sociopolitique de l'histoire de la diaspora noire tout en lui restituant son identité historique et en la faisant sortir de son invisibilité. Comme il le dit lui-même : " It is clear that having grown up in a world that didn't recognize me I understood - at a very early age - the importance of mapping the journey that had led to my lamentable state of invisibility. The path I would have to tread would be 'history' [...]. The task was to set about beating another - a different - path for myself which I, and perhaps others, might choose to follow" (166).

Cette voie suivie par Phillips lui a finalement permis de contrer le discours colonial d'autorité, de rendre visible l'invisible et audible l'indicible à travers son écriture historico-fictionnelle de la diaspora noire. La riche analyse de Françoise Clary nous invite, quant à elle, à découvrir tous les aspects de la transmission de la mémoire, du brouillage des frontières entre l'espace et le temps, à questionner l'écriture de l'altérité et nous guide avec justesse et précision vers une meilleure compréhension de la pensée et l'œuvre de Caryl Phillips. 
INDEX

Keywords : alterity, culture, diasporic consciousness, identity, memory, modernism, sociopolitics, transmission.

Mots-clés : altérité, culture, conscience diasporique, identité, mémoire, modernisme, narration historico-fictionnelle, sociopolitique, transmission

\section{AUTEURS}

\section{CHRISTINE DUALÉ}

Maître de conférences HDR

Université Toulouse Capitole

christine.duale@ut-capitole.fr 